\title{
AN OVERVIEW OF 3D TOPOLOGY FOR LADM-BASED OBJECTS
}

\author{
Nur Amalina Zulkifli ${ }^{\mathrm{a}^{*}}$, Alias Abdul Rahman ${ }^{\mathrm{a}}$ and Peter van Oosterom ${ }^{\mathrm{b}}$ \\ ${ }^{a}$ Department of Geoinformation, Faculty of Geoinformation and Real Estate, Universiti Teknologi Malaysia, UTM Skudai 81310, \\ Johor, Malaysia - (amalina.jc, alias.fksg)@gmail.com \\ ${ }^{\mathrm{b}}$ Section GIS technology, Department OTB, Faculty of Architecture and the Built Environment, TU Delft, The Netherlands - \\ P.J.M.vanOosterom@tudelft.nl
}

KEY WORDS: 3D topology, LADM, 3D cadastre, TEN

\begin{abstract}
:
This paper reviews 3D topology within Land Administration Domain Model (LADM) international standard. It is important to review characteristic of the different 3D topological models and to choose the most suitable model for certain applications. The characteristic of the different 3D topological models are based on several main aspects (e.g. space or plane partition, used primitives, constructive rules, orientation and explicit or implicit relationships). The most suitable 3D topological model depends on the type of application it is used for. There is no single 3D topology model best suitable for all types of applications. Therefore, it is very important to define the requirements of the 3D topology model. The context of this paper is a 3D topology for LADM-based objects.
\end{abstract}

\section{INTRODUCTION}

A lot of misunderstanding arises regarding the terms involved in cadastre (Bogaerts and Zevenbergen, 2001). In the past, most countries developed their own land administration systems (i.e. centralised or decentralised, deed registration or title registration, general boundaries or fixed boundaries). The misunderstandings between countries or organisations arise from a lack of shared concepts and terminology. Therefore, the concepts should be based on Land Administration Domain Model (LADM) international standard ISO 19152:2012, which also support for 3D representations. Although, it is still a relatively new standard (i.e. 'born' on $1^{\text {st }}$ December 2012), some countries already published works from various contexts of 3D cadastre based on LADM (i.e. the Netherlands, Russia, Australia, China and Indonesia). The purpose of the LADM is not to replace existing system, but rather to provide a formal language. The main reason to apply the LADM is to reuse the collective knowledge from many countries in land administration and to have unambiguous definitions of the key concepts.

Literature review on the LADM presented examples of various explorations in the application of the LADM (Karki et al., 2011; Ying et al., 2011; Vandysheva et al., 2012; Budisusanto et al., 2013). Initial steps have been taken by (Zulkifli et al., 2014) to develop a conceptual model of Malaysian country profile based on LADM. The presentation of country profile based on the standard is to understand the structure within the individual country land administration system and show examples of structures that can be useful in building profiles for other countries (Lemmen, 2012). Nevertheless, the Malaysian country profile only proposed 2D topology model and did not include 3D topology model for spatial units (but a pure geometry based approach). This will be reconsidered in this paper by exploring a 3D topology approach.

The Malaysian country profile used Winged Edge model for 2D topology representation (refer Figure 1). Topology boundaries do not intersect and do meet other boundaries at begin and end nodes. All topological boundaries are used once in positive and also once in negative direction. Unless the boundary is on the edge of the domain, then it is used only once. All associated boundaries together form one or more non-intersecting rings, defining at exactly one outer ring (with counter clockwise orientation) and one or more inner rings (with clockwise orientation). The 2D topology model is based on three primitives: node (in MY_Point class), edge (in MY_BoundaryFaceString class), and face (in MY_Lot2D class). Topology references (in MY_BoundaryFaceString class) are: edge $\rightarrow$ node (from node and to node), edge $\rightarrow$ edge (first right edge and last left edge), and edge $\rightarrow$ face (right face and left face). At the moment, the 2D topology model in Malaysian LADM country profile is not translated into physical model yet. It is important to test the model with real data set for validation purposes.

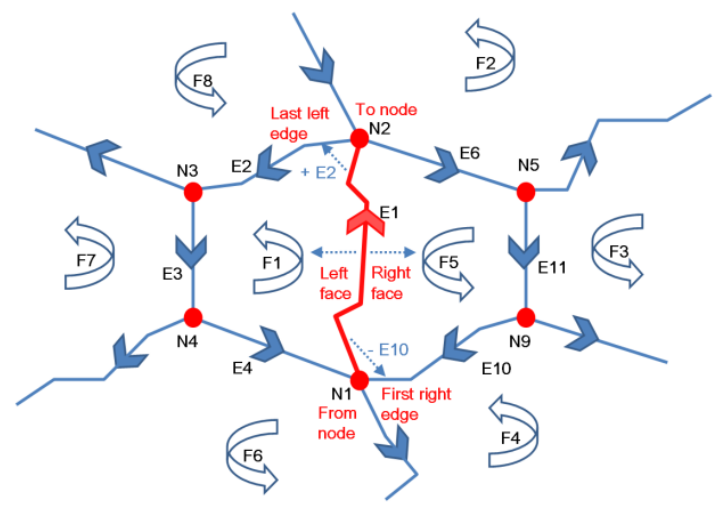

Figure 1. 2D topology model as used in the Malaysian country profile

Currently, 3D spatial units in the Malaysian country profile are based on pure geometry representation. There are several classes that store a 3D geometry attribute (i.e. GM_Solid) such as MY_Shared3DInfo and MY_Lot3D. MY_Shared3DInfo is a superclass of among others MY_Building and MY_Utility. Meanwhile, MY_Lot3D is a subclass of MY_GenericLot. Both MY_Shared3DInfo and MY_GenericLot are abstract classes (i.e. indicated with italic font) and do not have any instances. The 
geometry representation for 3D spatial unit will duplicate 'nodes' and 'edges' several times in database storage. It is not efficient, takes more time for data query, the redundancy has the danger of inconsistency, and the boundary primitives can not have their own attributes (e.g. such survey or quality information, often very important in land administration). Instead of giving each unit its own 3D geometry and duplicating the shared faces between neighbours, the potential of use topology need further investigation to represent various spatial units that share faces, which is often the case in large buildings with several units. Topological structures are important to represent object without redundancy. According to Molenaar (1998), topology and geometry aspects are modelled (in tables such as node, edge and face) separately from the objects.

\section{3D TOPOLOGY BASED ON LADM}

To model 3D topology, a number of 3D topological frameworks have been introduced from different research projects. These can be distinguished into two types of frameworks: 1 . classification of topological relationships between two objects, and 2. topological structures representing the structural relationship between many primitives and objects. Examples of the first type of framework (topological relationship between two objects) are the 9 Intersection Model (Egenhofer, 1995) and Dimensional-Extended Model (Billen et al., 2002). Further, in the context of the second type of framework (topological structures), several 3D topological models and approaches have been developed to construct a topologically correct datasets, e.g. (Penninga and van Oosterom, 2008), (Ledoux and Meijers, 2009), (Bormann and Rank, 2009), (Ghawana and Zlatanova, 2010), (Boguslawski et al., 2011) and Brugman et al., 2011). However, these models have not discussed on LADM standard. In Annex E7 of ISO 19152:2012 one example spatial profile is given for 3D topology structure. However, as indicated above more topological models exist and these should be further documented, refined, analysed and agreed on based on LADM standard.

The LADM provides conceptual descriptions for land administration, including 3D topology. The LADM also allows for organizing land related data in a standardized and interoperable way to support different types of spatial data. According to the requirements of LADM, topological information alone is not sufficient to describe a 3D spatial unit. Geometrical information must also be associated with each topological primitive; either direct geometries, or indirect (via related topological primitives with geometries). For 3D topology model in LADM as described in Spatial profiles of Annex E7, there are no overlapping volumes (3D_SpatialUnit). However, volumes may be open at the bottom or at the top, corresponding to non-bounded 3D_SpatialUnits (in this case, the size of the volume cannot be computed). Note that in 3D_Level, the attribute structure is fixed to '3D', and there still is an optional referencePoint, which should be provided via 3D GM_Point. There is a set of constraints defining a valid topological structure for a 3D volume partition. In case of the 3D topology representation, a 3D boundary has plus/minus orientation information included in the association to a $3 \mathrm{D}$ spatial unit (refer Figure 2).

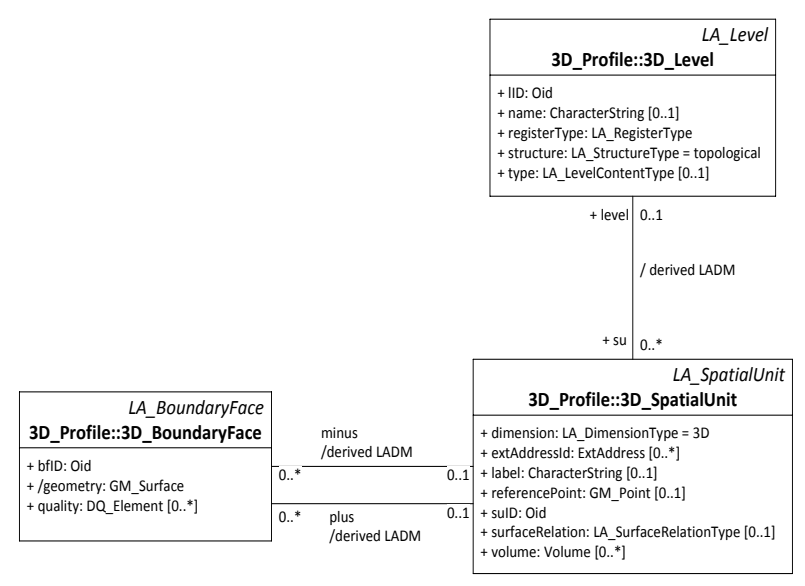

Figure 2. 3D topology based on LADM (after ISO 19152: 2012)

All topological boundary faces are used once in plus and also exactly once in minus direction. Unless the boundary face is on the edge of the domain, then either the plus or the minus direction is used once (and the other zero times). The boundary faces do not self-intersect and do meet other boundary faces at their boundaries. All 3D_BoundaryFaces have outward orientation (normal vector points to the outside). All the 3D_BoundaryFaces together form at least one outer shell and zero or more inner shells. In principle, the shells are closed, with the exception that they may open (unbound) to the top (sky) and bottom (earth) direction.

\section{CONCLUSION}

The LADM is an important standard for cadastral registration because it is being tested and practiced in an increasing number of countries (i.e. Karki et al., 2011; Vandysheva et al., 2012; Budisusanto et al., 2013; Zulkifli et al., 2014). Most of the literature review just proposes the conceptual model and do not include 3D topology structure representation. China has moved forward on 3D topology based on LADM by developing a prototype for cadastral registration (Ying et al., 2011). However, the body construction is using a closed polyhedron as 3D primitive. Further, the polyhedron approach might result in rather complex shapes and geometries (hard to validate). This can be avoided by using tetrahedron as 3D primitive, which limits the complexity of a single primitive (and adding primitives can then result in a rich variation of shapes again).

Based on the reviews of the 3D topological models, a very suitable 3D topology model is the approach based on a Tetrahedral Network (TEN), proposed by Penninga and van Oosterom (2008): the 'topological structure to organize tetrahedrons'. The TEN was selected due to its favourable characteristics from a computational point of view (i.e. only have four points). All surface elements of the TEN consist of flat faces, convex and they are all well defined, because the three points of each triangle always lie in the same plane. However, the TEN model need to be synchronized, described in a new spatial profile, with LADM specifications. The future work is to develop a conceptual model of the TEN based on LADM standard. Then, the proposed conceptual models (i.e. 2D and $3 \mathrm{D}$ topology) will be translated into physical model to develop a prototype for cadastral registration. 


\section{REFERENCES}

Billen R., S. Zlatanova, P. Mathonet and F. Boniver, 2002. The Dimensional Model: a framework to distinguish spatial relationships, in: Advances in Spatial Data handling, D.Richardson, P.van Oosterom (Eds.), Springer, pp. 285-298.

Boagearts, T. and Zevenbergen, J., 2001. Cadastral Systems Alternatives, In: Computers, Environment and Urban System, Theme Issue 'Cadastral System', p. 325-337, Volume 25, number 4-5, 2001, Elsevier Science, New York.

Boguslawski, P., Gold, C. M. and Ledoux, H., 2011. Modelling and analysing $3 \mathrm{~d}$ buildings with a primal/dual data structure. ISPRS Journal of Photogrammetry and Remote Sensing 66(2), pp. 188-197.

Borrmann, A. and Rank, E., 2009. Topological analysis of 3D building models using a spatial query language. Advanced Engineering Informatics 23(4), pp. 370-385.

Brugman, B., Tijssen, T. and van Oosterom, P., 2011. Validating a 3D topological structure of a 3D space partition. In: Advancing Geoinformation Science for a ChangingWorld, Springer, pp. 359-378.

Budisusanto, Y., Aditya, T. and Muryamto, R. 2013. LADM Implementation Prototype of 3D Cadastre Information System for Multi-Level Appartment in Indonesia. In proceedings of the $5^{\text {th }}$ Land Administration Domain Model Workshop, 24-25 September 2013, Kuala Lumpur, Malaysia, pp. 465-475.

Egenhofer, M. J., 1995. Topological relations in 3-D. Technical report, National Center for Geographic Information and Analysis and Deparment of Spatial Information Science and Engineering Department of Computer Science university of Maine.

Ghawana, T. and Zlatanova, S., 2010. Data consistency checks for building a 3D model: a case study of Technical University, Delft Campus, The Netherlands. Geospatial World (4). ISPRS Annals of the Photogrammetry, Remote Sensing and Spatial Information Sciences, Volume II-2/W1, ISPRS $8^{\text {th }}$ 3DGeoInfo Conference \& WG II/2 Workshop, 27 - 29 November 2013, Istanbul, Turkey.

ISO 2012. ISO 19152: 2012 Geographic Information - Land Administration Domain Model (LADM), Version 1 December 2012.

Karki, S., Thompson, R., McDougall, K., Cumerford, N. and van Oosterom, P. 2011. ISO Land Administration Domain Model and LandXML in the development of digital survey plan lodgement for 3D Cadastre in Australia. In proceedings of the $2^{\text {nd }}$ International Workshop on 3D Cadastre, 16-18 November 2011, Delft, The Netherlands, 20p.

Ledoux, H. and Meijers, M., 2009. Extruding building footprints to create topologically consistent $3 \mathrm{~d}$ city models. Urban and Regional Data Management, UDMS Annuals pp. $39-48$.

Lemmen, C.H.J. 2012. A Domain Model for Land Administration. PhD Thesis. Delft University of Technology, Delft, the Netherland.
Molenaar, M. 1998. An Introduction to the theory of spatial object modelling for GIS. Taylor and Francis.

Penninga, F. and van Oosterom, P.J.M. 2008. A Simplicial Complex-Based DBMS Approach to 3D Topographic Data Modelling, International Journal of Geographic Information Science, 22, 751-779.

Vandysheva, N., Sapelnikov, S., van Oosterom, P., De Vries, M., Spiering, B., Wouters, R., Hoogeveen, A. and Penkov, V. 2012. The 3D Cadastre Prototype and Pilot in the Russian Federation. In proceedings of the FIG Working Week 2012, Rome, Italy, $16 \mathrm{p}$.

Ying, S., Guo, R., Li, L., van Oosterom, P., Ledoux, H. and Stoter, J. 2011. Design and Development of a 3D Cadastral System Prototype based on the LADM and 3D Topology. In proceedings of the $2^{\text {nd }}$ International Workshop on 3D Cadastres, 16-18 November 2011, Delft, The Netherlands, pp.167-188.

Zulkifli, N.A., Abdul Rahman, A., Jamil, H., Teng C.H., Tan, L.C. and Looi, K.S., Chan K.L. and van Oosterom, P. 2014. Towards Malaysian LADM Country Profile for 2D and 3D Cadastral Registration System. In proceedings of the FIG Congress, 16-21 June 2014, Kuala Lumpur, Malaysia, 18p. 